\title{
Single-stage repair of extensive thoracic aortic aneurysms: Experience with the arch-first technique and bilateral anterior thoracotomy
}

\author{
Nicholas T. Kouchoukos, MD \\ Michael C. Mauney, MD \\ Paolo Masetti, MD \\ Catherine F. Castner, RN
}

From the Division of Cardiovascular and Thoracic Surgery, Missouri Baptist Medical Center, St Louis, Mo.

Read at the Eighty-fourth Annual Meeting of The American Association for Thoracic Surgery, Toronto, Ontario, Canada, April 25-28, 2004.

Received for publication April 23, 2004; revisions received June 4, 2004; accepted for publication June 16, 2004

Address for reprints: Nicholas T. Kouchoukos, MD, Cardiac, Thoracic and Vascular Surgery, Inc, 3009 N Ballas Rd, St Louis, MO 63131 (E-mail: ntkouch@aol.com).

J Thorac Cardiovasc Surg 2004;128:669-76 $0022-5223 / \$ 30.00$

Copyright (c) 2004 by The American Association for Thoracic Surgery

doi:10.1016/j.jtcvs.2004.06.037
Background: Staged procedures for extensive aneurysmal disease of the thoracic aorta are associated with a substantial cumulative mortality $(>20 \%)$ that includes hospital mortality for the 2 procedures and death (often from aortic rupture) in the interval between the 2 procedures. We have used a single-stage technique for operative repair of most or all of the thoracic aorta.

Methods: Forty-six patients with extensive disease of the thoracic aorta were managed with a single-stage procedure by using a bilateral anterior thoracotomy and transverse sternotomy, hypothermic circulatory arrest, and reperfusion of the aortic arch vessels first to minimize brain ischemia. Thirty-one patients with chronic, expanding type A aortic dissections had previous operations for acute type A dissection $(n=22)$, aortic valve repair or replacement $(n=4)$, coronary artery bypass grafting $(n=4)$, or no previous operation $(n=1)$. The remaining 15 patients had degenerative aneurysms $(n=12)$ or chronic type B dissections with proximal extension $(\mathrm{n}=3)$. The ascending aorta and aortic arch were replaced in all patients combined with resection of various lengths of descending aorta (proximal one third $[n=19]$, proximal two thirds to three quarters $[n=22]$, or all $[n=5])$. Coronary artery bypass grafting, valve replacement, or both were performed concomitantly in 19 patients.

Results: Hospital mortality was $6.5 \%$ (3 patients). Morbidity included reoperation for bleeding (17\%), mechanical ventilation for more than 72 hours $(42 \%)$, temporary tracheostomy (13\%), and temporary renal dialysis (9\%). No patient sustained a stroke. There have been 5 late deaths $(3,18,34,51$, and 79 months postoperatively) unrelated to the aortic disease. Four patients have undergone successful reoperation on the aorta (false aneurysm [n $=1]$, endocarditis $[\mathrm{n}=1]$, and progression of disease $[\mathrm{n}=2]$ ). Five-year survival was $75 \%$.

Conclusion: The single-stage, arch-first technique is a safe and suitable alternative to the 2-stage procedure for repair of extensive thoracic aortic disease.

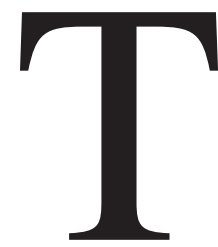

he optimal surgical technique for the treatment of extensive disease of the thoracic aorta is not clearly established. A staged approach has been favored by some surgical groups when the ascending aorta, the arch, and the descending thoracic or thoracoabdominal aorta are involved, and the elephant trunk technique has been widely used in this setting. ${ }^{1-4}$ However, recent reports with the elephant trunk technique have documented a cumulative mortality exceeding $20 \%$, which includes hospital mortality for the 2 procedures and death (commonly from aortic rupture) in the interval between the 2 procedures. ${ }^{3,5}$ Similarly, there is no clear consensus 


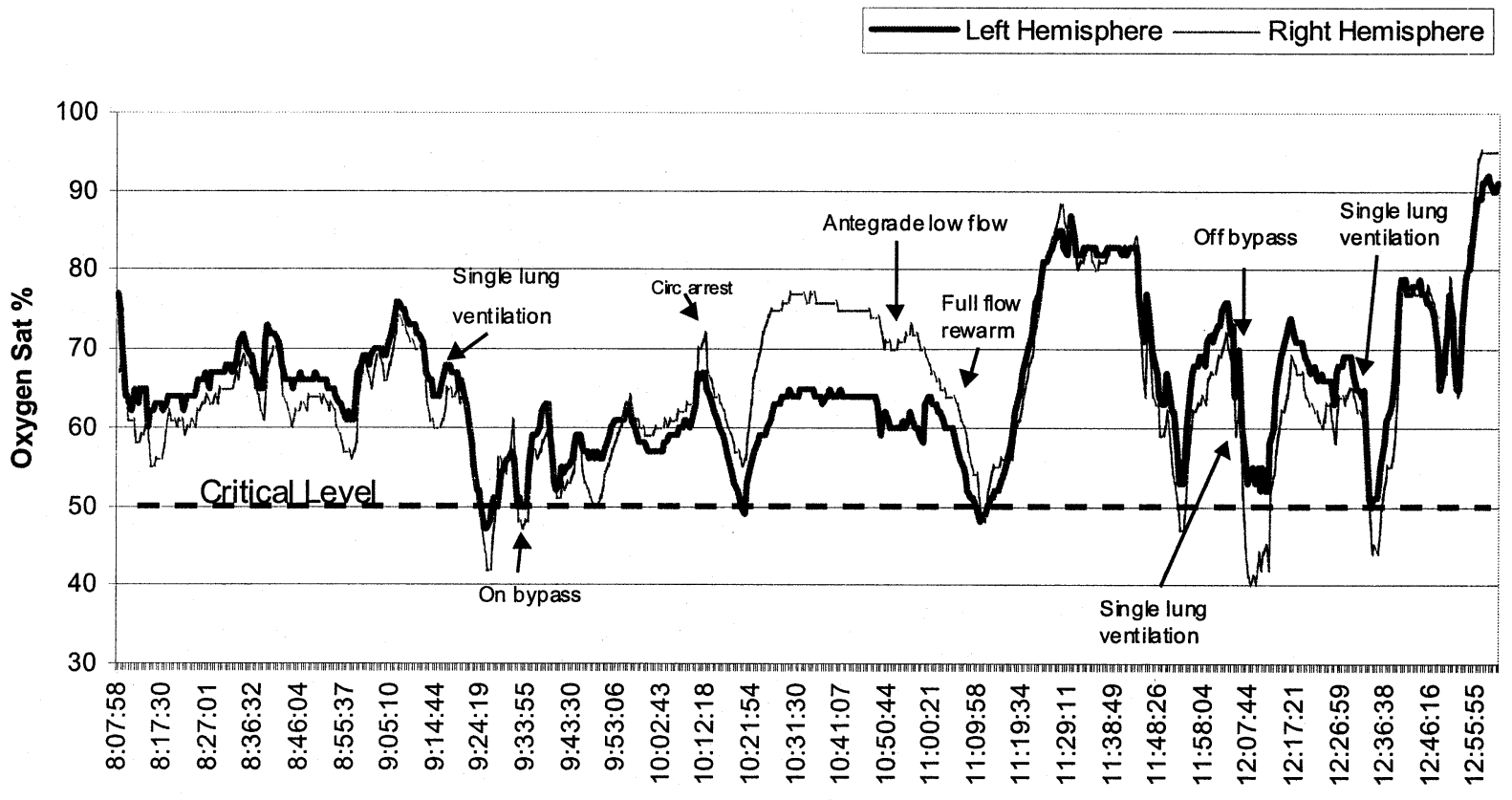

\begin{abstract}
Left baseline $\quad 64 \%$
Time

Critical level $\quad 51 \%$

Right baseline $61 \%$

Critical level $\quad 49 \%$

Figure 1. Continuous plot of left and right cerebral hemispheric oxygen saturation with the Somanetics Invos Cerebral Oximeter during aortic arch replacement with a branched graft and a brief (10 minute) interval of hypothermic circulatory arrest, followed by a 29 -minute interval of hypothermic brain perfusion through the right carotid and right vertebral arteries. During this period, oxygen saturation did not fall below the critical level $(80 \%$ of baseline). Similar findings during the intervals of circulatory arrest and hypothermic perfusion were noted in an additional 5 patients with the branched-graft technique in whom cerebral oxygen saturation was monitored.
\end{abstract}

regarding the optimal method of brain protection for procedures that involve total replacement of the aortic arch. Hypothermic circulatory arrest, antegrade brain perfusion, or retrograde brain perfusion, alone or in combination, have been used. ${ }^{6}$

In 1999, we reported our initial experience with singlestage repair of extensive disease of the thoracic aorta using a bilateral anterior thoracotomy (clamshell) incision and performance of the arch anastomosis first during a period of hypothermic circulatory arrest to minimize the duration of brain ischemia. ${ }^{7}$ In this report we present our updated experience with this technique.

\section{Methods}

\section{Patient Characteristics}

During a 9-year interval ending in January 2004, 46 consecutive patients underwent single-stage repair of the ascending aorta, the aortic arch, and varying lengths of the descending thoracic aorta with a bilateral anterior thoracotomy with transverse sternotomy incision and anastomosis of the arch vessels to the graft first. The mean age of the patients was 61 years (range, 30-81 years), and 33 (72\%) were men. Four (9\%) patients had Marfan syndrome. Thirty-three (72\%) patients had undergone previous operations on the thoracic aorta, the aortic valve, or the coronary arteries.

Thirty-one patients had chronic type A aortic dissections. Twenty-two of these patients had undergone previous ascending aortic replacement, with or without aortic valve replacement or repair, for acute type A aortic dissection and had progressive enlargement of the remaining dissected aortic arch and varying lengths of the descending thoracic aorta. The other 9 patients presented with chronic type A dissection after previous aortic valve replacement or repair (4 patients), coronary artery bypass grafting (4 patients), or without a previous operation (1 patient). Of the remaining 15 patients, 12 had extensive degenerative aneurysms, and 3 had chronic type B dissections with proximal extension. Three of the 12 patients with degenerative aneurysms had previous operations on the thoracic aorta. No patient in this series presented with acute dissection. The mean interval between the initial and the second procedure among the 33 patients undergoing reoperation was 69 months (range, 1-265 months).

The indications for operation were progressive enlargement of the involved aortic segments documented by means of computed tomography or magnetic resonance imaging in all patients and the presence of symptoms (chest pain, back pain, cardiac failure, and hoarseness) in $26(56 \%)$ patients. Rupture of the aneurysm was 
TABLE 1. Extent of descending thoracic aortic replacement and concomitant procedures

\begin{tabular}{lr}
\hline & $\begin{array}{r}\text { No. of } \\
\text { patients }\end{array}$ \\
\hline Extent of descending thoracic aortic replacement & \\
Proximal 1/3 & 19 \\
Proximal 2/3 to 3/4 & 22 \\
All & 5 \\
Concomitant procedures & 9 \\
Aortic root replacement & 7 \\
Aortic valve replacement & 7 \\
Coronary artery bypass grafting & 1 \\
Mitral valve replacement & 1 \\
Repair of sinus of Valsalva aneurysm & \\
\hline
\end{tabular}

present in 2 patients. All patients having elective operations underwent preoperative cardiac catheterization, assessment of pulmonary and renal function, and carotid duplex imaging. Other diagnostic studies (thoracic aortography, cerebral angiography, or computed tomographic or magnetic resonance imaging of the brain) were performed when indicated.

During the study interval, patients with aortic disease confined to the ascending aorta, the aortic arch, and the first few centimeters of the descending thoracic aorta were operated on through a median sternotomy. In one of these patients, a T extension through the third intercostal space was required to facilitate exposure of the descending thoracic aorta. Patients with aortic enlargement that extended below the diaphragm were managed with staged procedures.

\section{Operative Technique}

Our technique has been previously reported. ${ }^{7,8}$ In brief, it involves use of a bilateral anterior thoracotomy through the fourth intercostal space, transverse sternotomy, central or peripheral venous cannulation, and femoral or axillary artery cannulation for arterial return from the pump oxygenator. After establishing cardiopulmonary bypass, the left heart is vented, and when possible, a cannula is positioned in the coronary sinus for delivery of cold blood cardioplegic solution. During cooling, the ascending aorta is freed from the undersurface of the sternum, the left phrenic and left vagus nerves are isolated as a pedicle, and, after dividing the inferior pulmonary ligament of the left lung, the distal limit of the aortic disease is identified. When the appropriate nasopharyngeal and bladder temperatures are reached and the electroencephalogram becomes isoelectric, the head is packed in ice, circulatory arrest is established, and the ascending aorta is incised.

In the initial 35 patients, a cuff of aortic tissue was excised around the brachiocephalic arteries, and this cuff was sutured to an opening in the aortic graft opposite the site of a 10-mm sidearm graft during a period of circulatory arrest. As this anastomosis is being completed, cold $\left(16^{\circ} \mathrm{C}-18^{\circ} \mathrm{C}\right)$ blood is infused retrogradely into the superior vena caval cannula. ${ }^{9}$ After the anastomosis is completed, the aortic graft is clamped distal to the arch anastomosis, and the graft is filled with blood. Arterial flow is established through a second arterial line from the pump oxygenator that is attached to the sidearm graft or through the axillary artery if it is
TABLE 2. Perfusion data

\begin{tabular}{lcr}
\hline & Mean \pm SD & Range \\
\hline Cooling & $35 \pm 8$ & $24-50$ \\
Circulatory arrest & $32 \pm 14$ & $6-59$ \\
$\quad$ Aortic cuff technique $(\mathrm{n}=35)$ & $39 \pm 9$ & $23-59$ \\
$\quad$ Axillary artery perfusion with & $12 \pm 7$ & $6-29$ \\
$\quad$ branched graft technique $(\mathrm{n}=11)$ & & \\
Arch perfusion (hypothermic) & $30 \pm 8$ & $15-49$ \\
Spinal cord and lower body ischemia & $62 \pm 15$ & $20-90$ \\
Myocardial ischemia & $148 \pm 37$ & $69-237$ \\
Rewarming & $69 \pm 15$ & $35-110$ \\
Total cardiopulmonary bypass* & $177 \pm 48$ & $103-290$ \\
\hline
\end{tabular}

All values are given in minutes.

*Excludes duration of circulatory arrest.

used for arterial return. The aortic graft is then clamped proximal to the innominate artery, and antegrade perfusion of the brachiocephalic arteries is established at a flow rate of 800 to 1200 $\mathrm{mL} / \mathrm{min}$ and at a temperature of $20^{\circ} \mathrm{C}$ to $22^{\circ} \mathrm{C}$.

In the most recent 11 patients, a branched aortic graft and a technique to further reduce the duration of circulatory arrest of the brain were used. ${ }^{10}$ The axillary artery is used for arterial return from the pump oxygenator. An 8-mm or 10-mm collagen-impregnated polyester graft (Meadox Hemashield, Platinum Graft; Boston Scientific Inc, Natick, Mass) is anastomosed to the side of the axillary artery and connected to the arterial line. After establishing circulatory arrest, the aorta is opened and examined. The innominate, left carotid, and left subclavian arteries are transected at their origins. Flow through the axillary artery graft is re-established, and after evacuation of air from the 3 brachiocephalic arteries, they are clamped several centimeters from their origins, and perfusion of the brain through the right vertebral and right carotid arteries is initiated. The initial flow rates are between 10 and $15 \mathrm{~mL} \cdot \mathrm{kg}^{-1} \cdot \mathrm{min}^{-1}$ and are adjusted to maintain the mixed venous oxygen saturation measured in the right atrium at greater than $80 \%$. In addition, cerebral oxygen saturation was monitored in 6 patients over both cerebral hemispheres by using the Somanetics Invos Cerebral Oximeter (Somanetics Corp, Troy, Mich; Figure 1). The divided left subclavian, left carotid, and innominate arteries are sequentially anastomosed to the branches of an aortic branched graft (Meadox Hemashield, Platinum 4 Branch Graft; Boston Scientific Inc). Air is evacuated from the graft by releasing the clamps on the brachiocephalic arteries, distal and proximal clamps are placed on the aortic graft as previously described, and antegrade flow to the brain is established through the axillary artery graft.

After completion of the arch anastomosis by means of either of the above techniques, the anastomosis of the aortic graft to the distal aorta is completed at the appropriate level by using an open technique. The graft is stretched tightly to avoid buckling. Intercostal arteries above the T7-T8 level are ligated. Those below this level, if patent, are preserved by beveling the aorta to preserve the posterior wall. Hypothermic perfusion of the brain is continued during this interval. After completion of this anastomosis and evacuation of air from the graft and the distal aorta through temporary release of the aortic clamp distal to the left subclavian artery, flow is established in the antegrade direction from the axillary artery graft or from the $10-\mathrm{mm}$ sidearm of the aortic 
branched graft, and rewarming is initiated. During rewarming, aortic valve or aortic root replacement and coronary artery bypass grafting, if indicated, are performed. The proximal end of the aortic graft is sutured to the ascending aorta just above the level of the aortic commissures, to an existing ascending aortic graft, or to a newly inserted composite graft. Saphenous vein bypass grafts, if present, are anastomosed to the aortic graft. When rewarming is completed, air is removed from the heart and ascending aorta, and cardiopulmonary bypass is discontinued. The descending thoracic aorta is tightly wrapped around the graft.

\section{Extent of Resection and Concomitant Procedures}

Varying lengths of the ascending aorta and the entire aortic arch were resected and replaced in all patients. The extent of descending thoracic aortic replacement is shown in Table 1. For patients with aortic dissection, the descending thoracic aorta was transected at a level at which the diameter did not exceed 3 to $3.5 \mathrm{~cm}$. A portion of the septum between the true and false channels was excised, and the graft was sutured to the outer circumference of the aorta.

The concomitant procedures performed are shown in Table 1. They included aortic root, aortic valve, and mitral valve replacement; coronary artery bypass grafting; and repair of a sinus of Valsalva aneurysm.

\section{Perfusion Data}

A membrane oxygenator (Optima XP; Cobe Cardiovascular, Arvada, Colo) was used in all cases.

A common femoral artery was used for arterial return in 32 patients, and the right axillary artery was used in the remaining 14 patients. The mean durations of cooling, circulatory arrest, hypothermic arch perfusion, spinal cord and lower body ischemia, myocardial ischemia, rewarming, and cardiopulmonary bypass are shown in Table 2. Among the 35 patients in whom a cuff of aorta was used to attach the brachiocephalic arteries to the aortic graft, the mean duration of circulatory arrest was $39 \pm 9$ minutes, exceeded 50 minutes in 4 patients, and did not exceed 60 minutes in any patient. Among the 11 patients in whom axillary artery perfusion and a branched aortic graft were used, the mean duration of circulatory arrest of the brain was $12 \pm 7$ minutes, exceeded 13 minutes in only 2 patients, and did not exceed 30 minutes in any patient. This was followed by an interval of hypothermic $\left(20^{\circ} \mathrm{C}\right.$ $22^{\circ} \mathrm{C}$ ) perfusion of the brain from the right vertebral and right carotid arteries that averaged $34 \pm 8$ minutes. The duration of hypothermic arch perfusion for the entire group averaged $30 \pm 8$ minutes. The mean duration of lower body and spinal cord ischemia was $62 \pm 15$ minutes.

\section{Results}

\section{In-hospital Mortality}

There were 3 hospital deaths $(6.5 \% ; 70 \%$ confidence limits, $2.9 \%-12.7 \%$ ). One patient, a 71-year-old woman with an expanding chronic type A dissection and severe aortic regurgitation after a coronary artery bypass grafting procedure, died in the operating room of biventricular failure after thoracic aortic resection, aortic root replacement, and repeat coronary artery bypass grafting. A second patient, a 47- year-old man, died on the eighth postoperative day after a cardiac arrest. He underwent operation for acute type A aortic dissection 9 months previously and required reoperation for persisting severe aortic regurgitation and heart failure associated with progressive enlargement of the aortic arch and descending thoracic aorta. The third patient, a 71-year-old woman with a large, symptomatic, degenerative aneurysm and severe aortic regurgitation, died on the $23 \mathrm{rd}$ postoperative day of multiple organ system failure after replacement of the thoracic aorta and aortic valve replacement. She had paraplegia in the postoperative period.

\section{In-hospital Morbidity}

Reoperation for bleeding or for evacuation of the clot from the left pleural space was required in $8(17 \%)$ patients. The intraoperative mean transfusion requirements were 9 units of packed red cells, 8 units of fresh frozen plasma, 6 units of platelets, and 8.5 units of cryoprecipitate.

Mechanical ventilatory support for more than 72 hours was required in 19 (42\%) of the 45 operative survivors, and $6(13 \%)$ required temporary tracheostomy. The mean duration of ventilatory support was 9.4 days (median, 2.8 days; range, 1-91 days). All patients were weaned from mechanical ventilation. Five $(45 \%)$ of 11 patients in whom a branched aortic graft and limited circulatory arrest of the brain were used required mechanical ventilation for more than 72 hours, whereas $14(41 \%)$ of the 34 operative survivors in whom the aortic cuff technique and a longer interval of circulatory arrest were used required more than 72 hours of ventilatory support. Permanent left vocal cord paralysis, presumably resulting from stretching of the left vagus or the left recurrent laryngeal nerve occurred in 1 $(2.2 \%)$ patient.

Inotropic support with more than $5 \mu \mathrm{g} \cdot \mathrm{kg}^{-1} \cdot \mathrm{min}^{-1}$ of dobutamine for more than 24 hours was required in $5(12 \%)$ of the 45 operative survivors. One patient required intraoperative insertion of a right ventricular assist device for right ventricular dysfunction. This was removed on the third postoperative day, and the patient made a full recovery.

Renal failure requiring temporary ultrafiltration or hemodialysis occurred in $4(9 \%)$ patients. One of these patients died in the hospital. The remaining 3 patients had return of blood urea nitrogen and creatinine levels to baseline values before discharge from the hospital.

Permanent and transient neurologic dysfunction were evaluated according to the classification of Ergin and colleagues. ${ }^{11}$ No patient sustained a stroke. Transient neurologic dysfunction occurred in $6(13 \%)$ of the 45 operative survivors and resolved completely in all of them. It was present in $5(14 \%)$ of the 35 patients with the aortic cuff technique and 1 (9\%) of the 11 patients with the branchedgraft technique. 


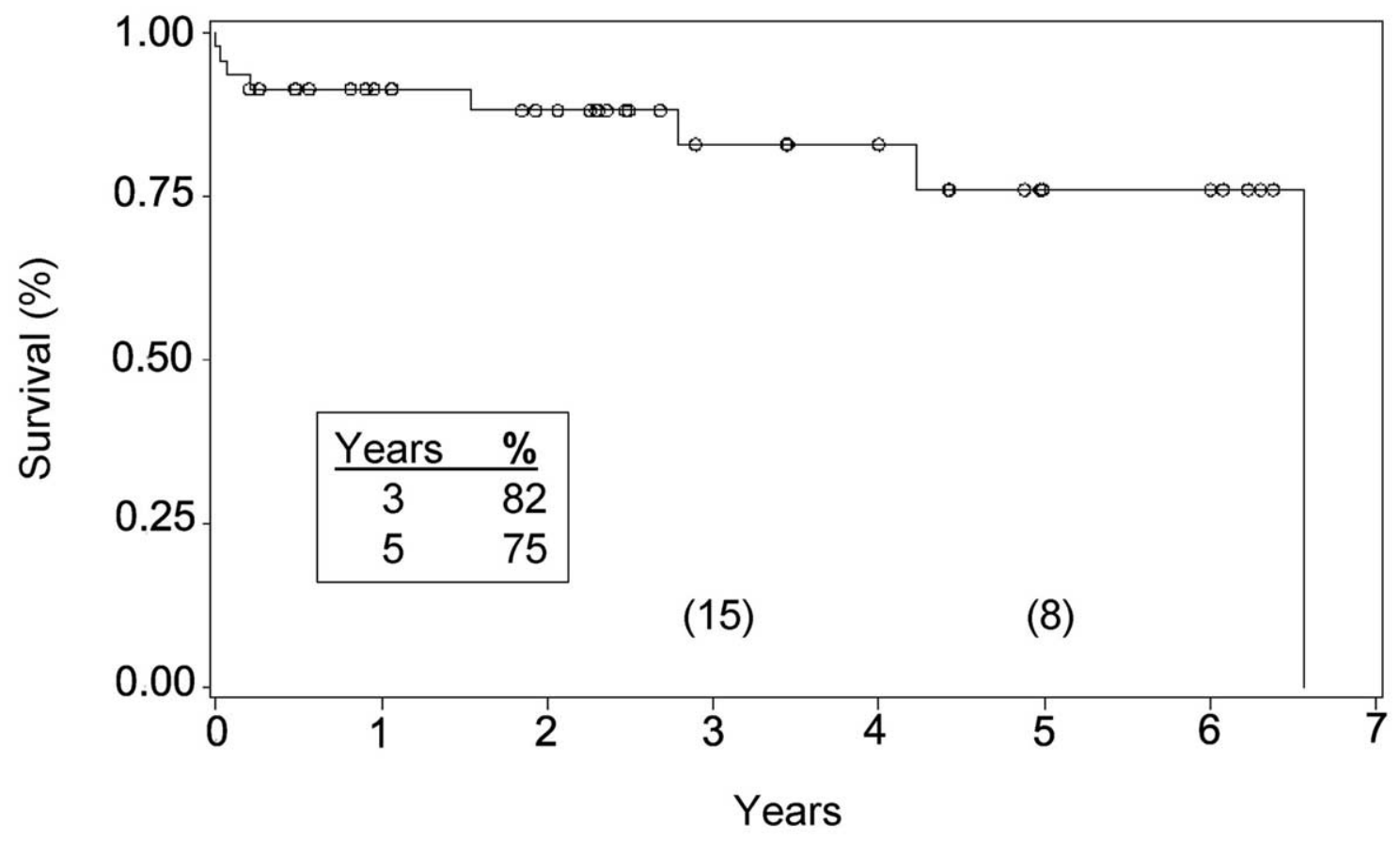

Figure 2. Product-limit estimate curve of survival after single-stage extensive replacement of the thoracic aorta. Numbers in parentheses indicate patients at risk.

There were no deep wound infections. One patient with chronic type A dissection required a femorofemoral artery bypass graft for postoperative occlusion of the left external iliac artery. One patient with Marfan syndrome required exploration of a groin incision for arterial bleeding and also a left thoracotomy to treat a persistent chylothorax. A third patient, also with Marfan syndrome, required laparotomy for intraperitoneal bleeding resulting from spontaneous rupture of a branch of the splenic artery. The mean duration of the postoperative hospital stay was 22 days (median, 12.5 days; range, 8-118 days).

\section{Late Mortality}

During the follow-up interval, which extends to 79 months, there have been 5 late deaths. These occurred 3, 18, 34, 51, and 79 months postoperatively from metastatic cancer, myocardial infarction, stroke, renal failure, and unknown cause, respectively. Follow-up information is available for all patients. Fifteen patients have been followed for more than 3 years and 8 for more than 5 years. The 3 -year and 5 -year survival rates are $82 \%$ and $75 \%$, respectively (Figure 2).

\section{Late Reoperations}

Four patients have undergone successful reoperations that involved the aortic graft ( 2 patients) or the remaining aorta (2 patients). One patient required reoperation on the aortic root and the ascending aortic graft for presumed infection
15 months postoperatively. The aortic root was replaced with an aortic allograft, and the ascending portion of the previously inserted aortic graft was replaced with a new polyester graft. A second patient had a false aneurysm surrounding the descending thoracic segment of the aortic graft 40 months postoperatively. At the time of the operation, erosion of the graft resulting from compression against a rib was noted, and a segment of the graft was replaced. The third patient, who had a chronic type B aortic dissection, required replacement of the remaining contiguous thoracic and upper abdominal aorta for aneurysmal enlargement 34 months after the initial operation. The fourth patient required resection of a separate infradiaphragmatic degenerative aneurysm 31 months postoperatively. All surviving patients have been followed with serial computed tomographic studies at 6- to 12-month intervals. To date, no other patient has had sufficient enlargement of the remaining thoracic aorta to require operation.

\section{Discussion}

Our extended experience with this single-stage technique for treatment of extensive thoracic aortic disease indicates that the early mortality is comparable with that reported with the first stage of a 2-stage approach by using the elephant trunk technique. ${ }^{1-4}$ Furthermore, the prevalence of early reoperation for bleeding, transfusion of blood components, prolonged mechanical ventilation, renal failure, tem- 
porary neurologic dysfunction, and stroke did not exceed that for the first stage of a 2-stage approach in a report by Safi and associates. ${ }^{2}$ Although our technique is not applicable to patients in whom the aneurysmal disease of the descending thoracic aorta is contiguous with disease of the abdominal aorta, it is a suitable alternative to staged procedures for disease confined to the thoracic aorta. Its use in the latter circumstance eliminates the risk of rupture of the remaining aneurysmal aorta in the interval between the first and second procedures, a common cause of death in this period, ${ }^{1-3}$ and the mortality and morbidity associated with a second thoracic aortic procedure.

The single-stage technique offers additional advantages over staged techniques and those that involve extensive resection of the descending thoracic aorta through a median sternotomy. The transverse bilateral anterior thoracotomy provides excellent exposure of the heart, the brachiocephalic arteries, the left phrenic and left vagus nerves, and the entire descending thoracic aorta. It avoids injury to the dilated ascending aorta that can occur with a median sternotomy incision, particularly during a reoperation, because the transverse thoracotomy incision is made below this level. The wide exposure of the left pleural space avoids excessive manipulation and compression of the left lung, which can result in intrapulmonary hemorrhage in a fully heparinized patient. If coronary artery bypass grafting is not required, mobilization of the heart from the pericardium is necessary only on the lateral surface of the right atrium and the adjacent interatrial groove. If coronary artery bypass grafting is necessary, exposure of the 3 major coronary arterial systems is easily accomplished. The aortic root is accessible if aortic valve or aortic root replacement is indicated. The right atrium and the interatrial groove are easily exposed if tricuspid or mitral valve procedures are required.

Use of a bilateral anterior thoracotomy provides excellent exposure of the aortic arch and the brachiocephalic arteries, thus permitting anastomosis of the graft to these vessels first and minimizing the duration of brain ischemia. The duration of circulatory arrest among the initial 35 patients in whom a cuff of aorta surrounding the brachiocephalic arteries was sutured to the aortic graft averaged 39 minutes and did not exceed 60 minutes in any patient. In the subsequent 11 patients, the mean duration of circulatory arrest of the brain was reduced to 12 minutes by use of the axillary artery for arterial return, a branched graft, and an interval of hypothermic brain perfusion from the right carotid and right vertebral arteries during which the 3 brachiocephalic vessels were attached to the branches of the aortic graft. Adequate blood flow to the left hemisphere was provided during this interval, as evidenced by adequate oxygen saturation levels measured by means of oximetry (Figure 1) and the absence of permanent postoperative neurologic deficits. Our experience with the latter technique in patients undergoing total aortic arch replacement with either a bilateral anterior thoracotomy or a median sternotomy has been previously reported, with similar results. ${ }^{10}$ It offers the additional advantages of providing arterial brain perfusion without the need for direct cannulation of the brachiocephalic arteries or a separate perfusion circuit. Furthermore, because the duration of hypothermic circulatory arrest and increasing age are important predictors of temporary neurologic dysfunction and of the persistence of fine-motor and memory deficits after operations on the aortic arch, reducing the duration of circulatory arrest should reduce the prevalence of these complications, particularly in elderly patients. ${ }^{12,13}$ Comparable results with hypothermic brain perfusion through the right brachial artery for aortic arch repair have been observed by Tasdemir and colleagues. ${ }^{14}$ They reported a hospital mortality of $7.6 \%$ and a prevalence of neurologic injury of $1.9 \%$ among 104 patients.

Potential disadvantages of the bilateral anterior thoracotomy include the sacrifice of both internal thoracic arteries and a high prevalence of pulmonary complications. However, no major wound complications were observed, and as noted previously, the prevalence of pulmonary complications did not exceed that reported for procedures that were performed with a median sternotomy approach. A low prevalence of pulmonary complications with a bilateral anterior thoracotomy for extensive thoracic aortic resections was observed by Doss and coworkers ${ }^{15}$ in 15 patients and by Minale and associates ${ }^{16}$ in 8 patients.

Reoperation for progression of aortic disease in the contiguous thoracic aorta has been required in only one of the 43 hospital survivors. Serial computed tomographic studies in the remaining 42 patients have not demonstrated substantial enlargement of the remaining thoracic aorta to date.

In summary, our extended experience with the singlestage, arch-first technique suggests that it is a safe and suitable alternative to the 2-stage procedure for repair of extensive aortic disease that is confined to the thoracic aorta. It might be of particular value in patients requiring reoperation for chronic type A aortic dissection who have substantial dilatation of the proximal descending thoracic aorta that might preclude safe suturing of an aortic graft to this segment. Our current technique for brain protection with axillary artery cannulation, a brief period of circulatory arrest, subsequent hypothermic antegrade brain perfusion, and a branched graft has substantially reduced the duration of circulatory arrest of the brain. It obviates the need for cannulation of the brachiocephalic arteries and the attendant risk of embolization and appears safe, as evidenced by the absence of stroke and a low prevalence of temporary neurologic dysfunction. 


\section{References}

1. Heinemann MK, Buehner B, Jurmann MJ, Borst H-G. Use of the "elephant trunk technique" in aortic surgery. Ann Thorac Surg. 1995; 60:2-7.

2. Safi HJ, Miller CC III, Estrera AL, et al. Staged repair of extensive aortic aneurysms. Morbidity and mortality in the elephant trunk technique. Circulation. 2001;104:2938-42.

3. Schepens MA, Dossche KM, Morshuis WJ, van den Barselaar PJ, Heijmen RH, Vermeulen FE. The elephant trunk technique: operative results in 100 consecutive patients. Eur J Cardiothorac Surg. 2002; 21:276-81.

4. Kieffer E, Koskas F, Godet G, et al. Treatment of aortic arch dissection using the elephant trunk technique. Ann Vasc Surg. 2000;14:612-9.

5. Estrera AL, Miller CC III, Porat EE, Huynh TTT, Winnerkvist A, Safi HJ. Staged repair of extensive aortic aneurysms. Ann Thorac Surg. 2002;74(suppl):S1803-5.

6. Griepp RB. Cerebral protection during aortic arch surgery. J Thorac Cardiovasc Surg. 2001;121:425-7.

7. Rokkas C, Kouchoukos NT. Single-stage extensive replacement of the thoracic aorta: the arch-first technique. J Thorac Cardiovasc Surg. 1999;117:99-105.

8. Kouchoukos NT, Masetti P, Rokkas C, Murphy SF. Single-stage reoperative repair of chronic type A aortic dissection by means of the arch-first technique. J Thorac Cardiovasc Surg. 2001;122:578-82.

9. Kouchoukos NT. Adjuncts to reduce the incidence of embolic brain injury during operations on the aortic arch. Ann Thorac Surg. 1994; 57:243-5.

10. Kouchoukos NT, Masetti P. Total aortic arch replacement with a branched graft and limited circulatory arrest of the brain. J Thorac Cardiovasc Surg. 2004;128:233-7.

11. Ergin MA, Uysal S, Reich DL, et al. Temporary neurological dysfunction after deep hypothermic circulatory arrest: a clinical marker of long-term functional deficit. Ann Thorac Surg. 1999;67:1887-90.

12. Ergin MA, Galla JD, Lansman SL, Quintana C, Bodian C, Griepp RB. Hypothermic circulatory arrest in operations on the thoracic aorta. J Thorac Cardiovasc Surg. 1994;107:788-99.

13. Reich DL, Uysal S, Sliwinski M, et al. Neuropsychologic outcome after deep hypothermic circulatory arrest in adults. J Thorac Cardiovasc Surg. 1994;117:156-63.

14. Tasdemir O, Saritas A, Kucuker S, Ozatik MA, Sener E. Aortic arch repair with right brachial artery perfusion. Ann Thorac Surg. 2002;73: 1837-42.

15. Doss M, Woehleke T, Wood JP. The clamshell approach for the treatment of extensive thoracic aortic disease. J Thorac Cardiovasc Surg. 2003;126:814-7.

16. Minale C, Splittgerber FH, Wendt G, Messmer BJ. One-stage intrathoracic repair of extended aortic aneurysms. J Card Surg. 1994;9:60413.

\section{Discussion}

Dr R. Scott Mitchell (Stanford, Calif). Dr Kouchoukos and his colleagues are to be congratulated on a courageous series of very difficult patients, and these are very difficult patients, as you saw from that one angiogram. We are also seeing an increasing number of these challenging patients, many of whom have survived the acute repair of their type $\mathrm{A}$ dissections and now present with continued late dilation of their dissected arch and descending thoracic aorta. Patients such as this comprised more than two thirds of this series.

In the initial 35 patients, hypothermic arrest with retrograde cerebral perfusion was used, and antegrade perfusion was used in the most recent 11 patients. Operative mortality was commendable at $6.5 \%$, and stroke was absent, with neurologic deficits being similarly low.

However, several morbid events did occur, primarily respiratory in nature, with $42 \%$ requiring rather prolonged mechanical ventilation, and $13 \%$ requiring tracheostomy. And therefore although I agree with the major details, primarily antegrade cerebral perfusion and use of the 4 branched-graft, arch-first emphasis, clearly this extensive operation exacts a significant toll.

After experience with about a dozen of these patients in my own hands, particularly one with a patent thoracic artery that was in my face for the entire day, we have come to prefer a 2-stage repair. I think the first stage of this, placing an elephant trunk, doing whatever arch work you need, and then the ascending, can be accomplished with similar mortality but perhaps less morbidity.

Dr Safi has given us a pretty good estimate of the interval of mortality. It is about $2 \%$ while you are waiting, and then the third stage, using stent graft technology, might be significantly better.

Therefore with this in mind, I have several questions. First, do you have an estimate of the respiratory reserve necessary to allow a patient to survive this procedure?

Dr Kouchoukos. All but 2 of the 46 patients were operated on electively and they had pulmonary function tests and evaluation by a pulmonologist preoperatively. None of these 46 patients were considered inoperable on the basis of these evaluations.

Dr Mitchell. What kind of clinical guidelines do you use?

Dr Kouchoukos. We did not employ any specific clinical guidelines to determine operability. Although pulmonary complications occurred commonly in the postoperative period, none of the early or late deaths were the result of respiratory failure and all patients were weaned from mechanical ventilation.

Dr Mitchell. Have you operated in the face of a patent thoracic artery, and is that a relative contraindication? How do you manage that?

Dr Kouchoukos. We have operated on patients with a patent left internal thoracic artery graft to the anterior descending coronary artery. The transverse thoracotomy permits identification, isolation, and protection of the thoracic artery pedicle.

Dr Mitchell. Your cerebral oximetry is fascinating. We typically put a perfusion balloon in the left carotid artery. With your perfusion data, do you think that is totally necessary?

Dr Kouchoukos. Using right axillary artery cannulation, hypothermic $\left(20^{\circ} \mathrm{C}-22^{\circ} \mathrm{C}\right)$ perfusion, packing of the head in ice, and, in the most recent cases, continuous monitoring of cerebral oxygen saturation, we do not believe it is necessary to perfuse the left carotid directly. We also keep the mixed venous oxygen saturation measured in the right atrium above $80 \%$ by adjustment of the axillary artery perfusion pressure and flow.

Dr Mitchell. Some patients sort of have a cervical arch in which the whole arch migrates up into the apex of the left chest. Is that accessible still with this technique?

Dr Kouchoukos. With the bilateral anterior thoracotomy and transverse sternotomy, exposure of the aortic arch is excellent, even when the brachiocephalic arteries are displaced into the apex of the left hemithorax. Difficulty in exposing such displaced vessels through a median sternotomy was one of the reasons we switched to the bilateral anterior thoracotomy.

Dr Richard J. Shemin (Boston, Mass). This is an amazing series, but looking to the future, I wonder what your opinion would be about doing a classical elephant trunk but shortening the time interval to repairing the residual dissection in the descending aorta with an extension stent graft attached to the elephant trunk. In that way you avoid the second operation. I would speculate that the 
stenting could be done very soon after the more classic primary approach.

Dr Kouchoukos. Use of an endograft in the descending thoracic aorta as an extension of the elephant trunk procedure is certainly an option. If the aortic disease is degenerative, where the upper thoracic intercostal arteries are small and often occluded by thrombus, an endograft might be a suitable alternative to the technique we have described. However, with chronic dissection, which was present in 34 of the 46 patients, most of the intercostal arteries are patent and backbleed vigorously, and I would be concerned about the potential for major endoleaks. In addition, the true lumen is often severely compressed by the expanding false lumen and it would be difficult to insert a suitable sized endograft into the true lumen.

Dr G. Hossein Almassi (Milwaukee, Wis). Dr Kouchoukos, I noticed that your oldest patient was 81 years old. Is there an age limit beyond which you would turn a patient down for this operation, such as 85 or 90 years? We seem to be seeing older patients presenting with this extensive aneurysmal disease.

Dr Kouchoukos. We have not established an age limit above which we would not recommend operation. Our oldest patient, whom you referred to, had a large, expanding, symptomatic chronic dissecting aneurysm but was otherwise in good health. She died 51 months postoperatively of renal failure. Careful risk assessment is obviously important, but to date no patient has been denied operation who could tolerate either a median sternotomy or a left thoracotomy incision.

Dr Robert A. Dion (Leiden, The Netherlands). Dr Kouchoukos, could you just elaborate a bit more on the approach-which intercostal space you are opening - on both sides, and is it a completely symmetric thoracotomy on both sides?

Dr Kouchoukos. Unfortunately, the time constraints on my presentation did not permit an adequate description of the operative technique. We use a bilateral fourth intercostal space incision and divide the sternum transversely. The incision is extended to the anterior axillary line on the right and to the mid or posterior axillary line on the left. If the procedure is a reoperation and coronary artery bypass grafting is not required, the pericardium is only incised over the right atrium to permit insertion of a left heart vent through the right superior pulmonary vein, a cardioplegia cannula into the coronary sinus, and a cannula for venous return. We frequently use a 2-stage cannula inserted into a femoral vein, which eliminates the need for a venous cannula in the operative field.

\section{JTCVS On-Line Manuscript Submission and Review Please visit http://www.editorialmanager.com/jtcvs/}

Effective September 15, 2001, authors and reviewers may submit manuscripts and reviews electronically via Editorial Manager, our new Web-based system with full electronic submission, review, and status update capabilities.

As we move from paper to electronic submissions, the Editorial Office will make proxy submissions of all manuscripts accompanied by a diskette containing the electronic files of the text, tables, and figures. Editors, authors, and reviewers will receive automatic e-mails when significant events occur.

We strongly encourage all authors and reviewers to use Editorial Manager. Although we will continue to accommodate the submission of paper manuscripts for some months, our goal is to be completely electronic within 9 to 12 months.

All individuals currently in our database for whom we have e-mail addresses will receive via e-mail a system-assigned username and password that can be used to log in to the system without prior registration. All those not receiving the e-mail must register the first time they use the system.

As with any broad systemic change, the conversion to the new system will take some time to complete. We ask your patience as we replace our in-office database with the new system. We also encourage you to take advantage of the speed and efficiency that the new system will provide for us all: editor, author, reviewer, and publisher. 